OPEN ACCESS

Edited by:

Akihide Ryo,

Yokohama City University, Japan

Reviewed by:

Takatoki Yamamoto,

Tokyo Institure of Technology, Japan Keiichiro Kushiro,

The University of Tokyo, Japan

Ryuichi Sugiyama,

Yokohama City University School of

Medicine, Japan

*Correspondence:

Gaku Imamura

imamura.gaku@nims.go.jp

Specialty section:

This article was submitted to

Virology,

a section of the journal

Frontiers in Microbiology

Received: 22 January 2016 Accepted: 24 March 2016

Published: 14 April 2016

Citation:

Imamura G, Shiba K and Yoshikawa G (2016) Finite Element Analysis on Nanomechanical Detection of Small

Particles: Toward Virus Detection.

Front. Microbiol. 7:488

doi: 10.3389/fmicb.2016.00488

\section{Finite Element Analysis on Nanomechanical Detection of Small Particles: Toward Virus Detection}

\author{
Gaku Imamura ${ }^{1 *}$, Kota Shiba ${ }^{1,2}$ and Genki Yoshikawa ${ }^{1}$ \\ ${ }^{1}$ World Premier International Research Center Initiative, International Center for Materials Nanoarchitectonics, National \\ Institute for Materials Science, Tsukuba, Japan, ${ }^{2}$ International Center for Young Scientists, National Institute for Materials \\ Science, Tsukuba, Japan
}

Detection of small particles, including viruses and particulate matter (PM), has been attracting much attention in light of increasing need for environmental monitoring. Owing to their high versatility, a nanomechanical sensor is one of the most promising sensors which can be adapted to various monitoring systems. In this study, we present an optimization strategy to efficiently detect small particles with nanomechanical sensors. Adsorption of particles on the receptor layer of nanomechanical sensors and the resultant signal are analyzed using finite element analysis (FEA). We investigate the effect of structural parameters (e.g., adsorption position and embedded depth of a particle and thickness of the receptor layer) and elastic properties of the receptor layer (e.g., Young's modulus and Poisson's ratio) on the sensitivity. It is found that a membrane-type surface stress sensors (MSS) has the potential for robust detection of small particles.

Keywords: nanomechanical sensors, cantilever sensors, membrane-type surface stress sensors (MSS), finite element analysis (FEA), virus detection

\section{INTRODUCTION}

Nanomechanical sensors have been attracting great attention because of their versatility. For example, they can detect diverse chemical species ranging from gaseous to biological molecules, including volatile organic compounds (VOCs), DNA, and proteins (Barnes et al., 1994; Gimzewski et al., 1994; Thundat et al., 1994; Buchapudi et al., 2011). Detection of bioorganisms such as viruses and bacteria has also been reported (Buchapudi et al., 2011). Since the first demonstration of nanomechanical sensing in 1994 (Gimzewski et al., 1994), cantilever-type sensors with optical readouts have been widely used. In 2011, a membrane-type surface stress sensor (MSS) was developed and achieved both high sensitivity and small size by means of structural optimization with chipintegrated piezoresistive read-out (Yoshikawa et al., 2011). This MSS platform also demonstrated the detection of proteins using a simple dipping system that is compatible with a standard 96-well plate for practical assays (Hosokawa et al., 2014).

Owing to these advantages, nanomechanical sensors are expected to be a key technology to environmental monitoring systems. In addition to toxic chemical species in air, there is a great need for detection of airborne particles including dust, particulate matter (PM), and viruses (Aliabadi et al., 2011; Després et al., 2012; Nemmar et al., 2013; Kim et al., 2015). As nanomechanical sensors can recognize such particles by detecting mechanical properties, it is important to understand the mechanics of the nanomechanical sensing system: Adsorption of particles on the receptor layer and the stress and deformation caused by adsorbed particles. 
In the present study, we investigate the nanomechanical detection of particles using finite element analysis (FEA). We focus on the two types of nanomechanical sensors; cantilevertype sensors and MSS, and explore an optimized structure for the efficient detection of particles through the mechanical stress induced in the receptor layer. We also discuss the perspective on virus detection using these nanomechanical sensors.

\section{MATERIALS AND METHODS}

Analytical solutions of nanomechanical sensing are available for a simple cantilever model. For example, the deflection of the freeend of a cantilever $(\Delta z)$ induced by isotropic internal strain in a receptor layer $\left(\varepsilon_{f}\right)$ is given by the following equation (Yoshikawa, 2011):

$$
\begin{gathered}
\Delta z=\frac{3 l^{2}\left(t_{f}+t_{c}\right)}{(A+4) t_{f}^{2}+\left(A^{-1}+4\right) t_{c}^{2}+6 t_{f} t_{c}} \varepsilon_{f}, \\
A=\frac{E_{f} w_{f} t_{f}\left(1-v_{c}\right)}{E_{c} w_{c} t_{c}\left(1-v_{f}\right)},
\end{gathered}
$$

where $l$ is the length of a cantilever, and $E_{f}\left(E_{c}\right), v_{f}\left(v_{c}\right)$, and $t_{f}\left(t_{c}\right)$ are the Young's modulus, the Poisson's ratio, and the thickness of a receptor layer (a cantilever), and $w_{c}$ and $w_{f}$ are the width

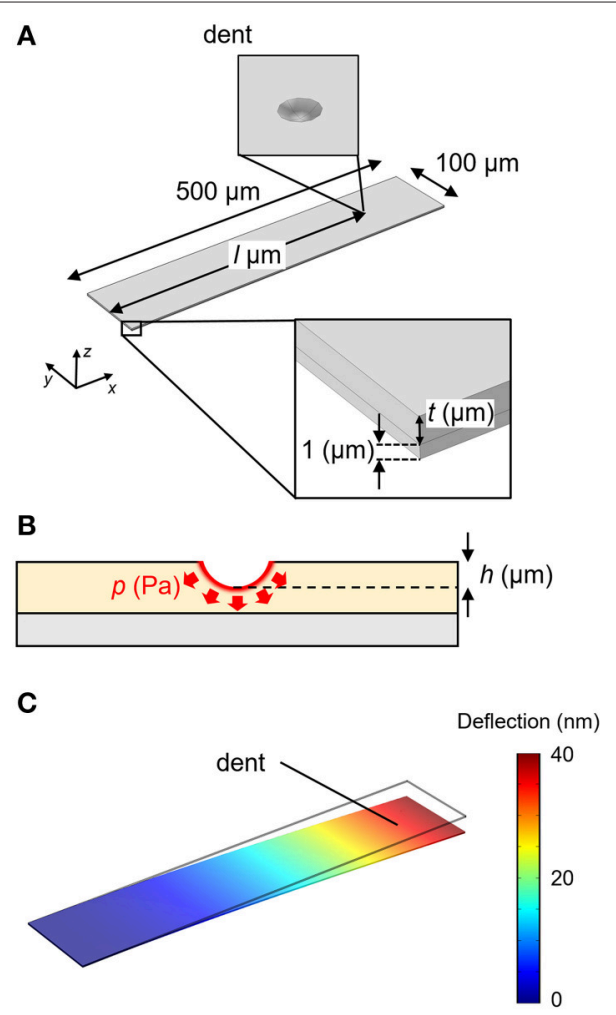

FIGURE 1 | (A) Structure of the cantilever-type sensor. (B) Cross sectional image of the cantilever at the dent. (C) Result of the FEA simulation for the cantilever-type sensor. The distribution of displacement is plotted as a color gradient. of a cantilever and a receptor layer, respectively. If a cantilever is covered with a very thin receptor layer $\left(t_{c} \gg t_{f}\right.$ and $\left.w_{c}=w_{f}\right)$, Equation (1) reduces to the following equation, which is known as the Stoney's equation (Stoney, 1909):

$$
\Delta z=\frac{3\left(1-v_{c}\right) l^{2}}{E_{c} t_{c}^{2}} \sigma_{\text {surf }},
$$

where $\sigma_{\text {surf }}$ is the surface stress defined as $\sigma_{\text {surf }}=\sigma_{f} \cdot t_{f}$, and $\sigma_{f}=\varepsilon_{f} \cdot E_{f} /\left(1-v_{f}\right)$. However, the application of these models is limited to analytically simple problems. To investigate more complex systems, FEA is an effective option, providing numerical solutions. Since the adsorption of particles on a solid receptor layer with three dimensional stress distribution is too complicated to be analytically modeled, we employed FEA in the present study.

FEA simulations were performed in COMSOL Multiphysics 5.1 ${ }^{\circledR}$ with the Structural Mechanics module. Each structure was meshed with 20,000 60,000 elements, which give sufficient resolution for the present simulations. We investigated the deflection of a silicon cantilever with dimensions $500 \times 100 \times$ $1 \mu \mathrm{m}$ as shown in Figure 1A. A fixed constraint was applied on one end (fixed-end). The receptor layer with a thickness of $t \mu \mathrm{m}$ is coated on the cantilever. A particle with a radius of $0.1 \mu \mathrm{m}$ is embedded in the receptor layer with a depth of $h \mu \mathrm{m}$, applying a constant pressure $p \mathrm{~Pa}$ at the interface (Figure 1B). To simulate such a situation, we set a dent on the receptor layer with a boundary load of $p \mathrm{~Pa}$ at the surface. The dent is located
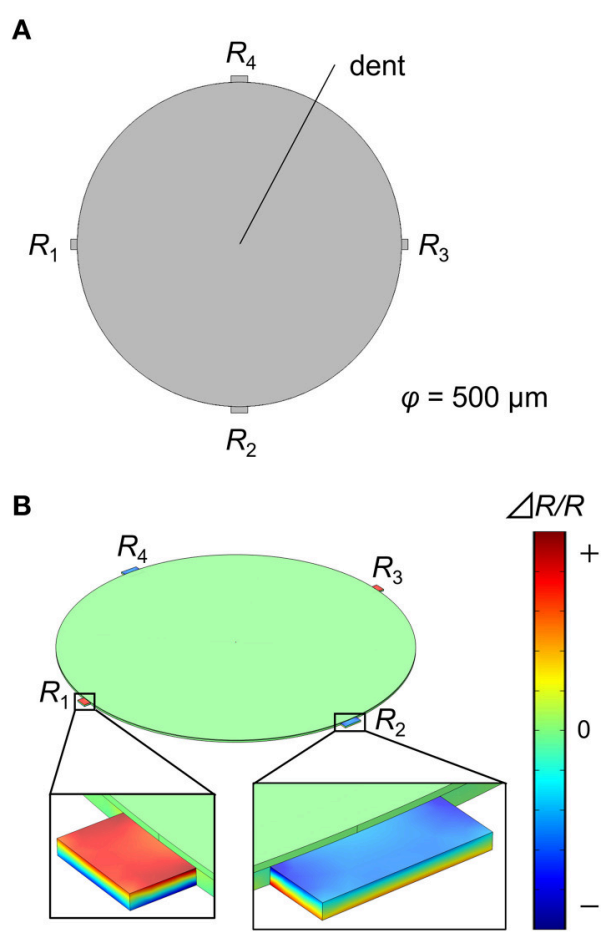

FIGURE 2 | (A) Structure of the MSS. (B) Result of the FEA simulation for the MSS. The distribution of relative resistance change is plotted as a color gradient. 
at $l \mu \mathrm{m}$ from the fixed-end. We investigated the deflection; the displacement of the free-end of the cantilever (Figure 1C). In the case of MSS, the diameter and the thickness of the membrane are set at 500 and $2.5 \mu \mathrm{m}$, respectively, with a $t-\mu \mathrm{m}$ thick receptor layer on it (Figure 2A). The dent is also placed on the receptor layer (default position is set at the center) to simulate the adsorption of a particle. We investigated the change in the relative resistance $\left(\Delta R_{\text {tot }} / R_{\text {tot }}\right)$ of a full Wheatstone bridge in an MSS composed of four resistors embedded in the sensing beams, $R_{1}-R_{4}$ (Figure 2B). The sensing signal, $V_{\text {out }}$ is approximately described as:

$$
V_{\text {out }}=\frac{V_{B}}{4}\left(\frac{\Delta R_{1}}{R_{1}}-\frac{\Delta R_{2}}{R_{2}}+\frac{\Delta R_{3}}{R_{3}}-\frac{\Delta R_{4}}{R_{4}}\right)=\frac{V_{B}}{4}\left(\frac{\Delta R_{\text {tot }}}{R_{\text {tot }}}\right),
$$

where $V_{B}$ is the bridge voltage, and $\Delta R_{i}$ is the change in the resistance of $R_{i}$ (Yoshikawa et al., 2011). A fixed constraint was applied on the end of each beam.

\section{RESULTS}

\section{Cantilever-Type Sensor}

The dependence on the applied pressure, $p$, is investigated. It is clear that the deflection of the cantilever is exactly proportional to $p$ over a wide range (Figure $\mathbf{3 A}$ ). This result suggests that the dependence of the deflection on the other parameters is not affected by $p$. We confirmed this condition by investigating the dependence on the other parameters while varying $p$. Figures 3B,C show the dependence on $l$, showing a natural consequence of simple mechanics: a particle adsorbed near the end of the cantilever leads to a larger deflection. The depth of the dent which corresponds to the embedded depth of a particle strongly affects the deflection. Figure 3D shows the deflection as a function of dent depth $h$ with a nonlinear relationship. Since the data in Figure 3D can be fitted well with the quadratic function, the deflection is found to be related with the projected area of the dent $s\left[s=\pi\left(2 r h-h^{2}\right)\right.$, where $r$ is a radius of a particle], rather than the surface area of the dent $S(S=2 \pi r h)$.

Figures 4A,B show the effects of Young's modulus $E$ and Poisson's ratio $v$, respectively. It has been found that the deflection is independent of $v$, while $E$ significantly affects the deflection. The deflection exhibits little dependence on $E$ below $10^{8} \mathrm{~Pa}$. However, a higher Young's modulus yields a lower deflection when $E$ is larger than $10^{9} \mathrm{~Pa}$. The thickness dependence is summarized in Figure 4C. It clearly shows that the thickness dependence is significantly affected by Young's modulus. The deflection decreases with increasing thickness, and it becomes drastic with $E>10^{7} \mathrm{~Pa}$.

\section{MSS}

The effects of applied pressure $p$, position, and dent depth $h$ on $\Delta R_{\text {tot }} / R_{\text {tot }}$ were investigated for MSS. Figure $5 \mathrm{~A}$ depicts

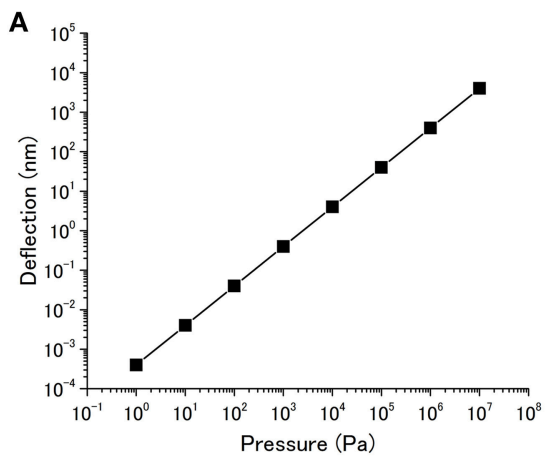

C

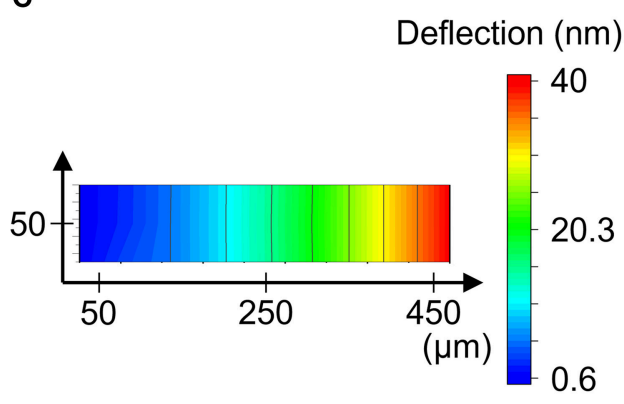

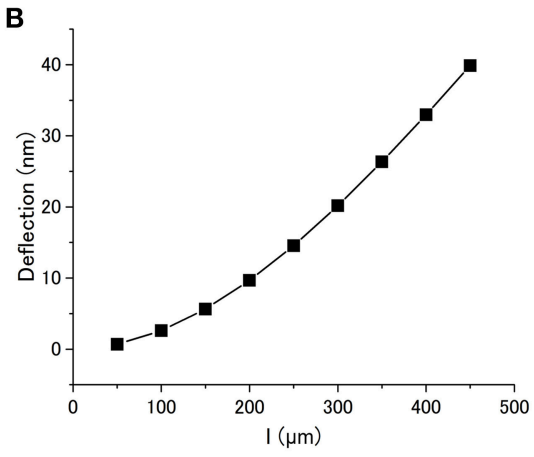

D

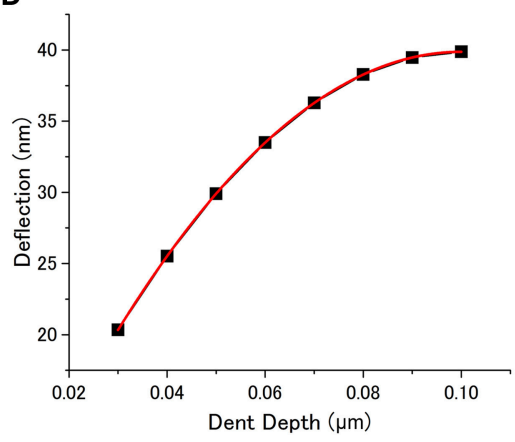

FIGURE 3 | (A) Dependence of the deflection on the applied pressure $\left(=450 \mu \mathrm{m}, h=0.1 \mu \mathrm{m}, t=1 \mu \mathrm{m}, E=1 \times 10^{9} \mathrm{~Pa}, v=0.4\right)$. (B) Dependence of the deflection on the position of the dent (distance from the fixed end). (C) Dependence of the deflection on the position of the dent. The resultant cantilever deflection is plotted as a color gradient $\left(p=1 \times 10^{5} \mathrm{~Pa}, h=0.1 \mu \mathrm{m}, t=1 \mu \mathrm{m}, E=1 \times 10^{9} \mathrm{~Pa}, v=0.4\right)$. (D) Dependence of the deflection on the dent depth $\left(p=1 \times 10^{5} \mathrm{~Pa}, I=450 \mu \mathrm{m}\right.$, $t=1 \mu \mathrm{m}, E=1 \times 10^{9} \mathrm{~Pa}, v=0.4$ ). The plot is fitted with a quadratic function (red curve). 
$\Delta R_{\text {tot }} / R_{\text {tot }}$ as a function of $p$, showing a linear relationship over a wide range. Same as a cantilever-type sensor, variation in $p$ is found not to affect the dependence on other parameters in the case of MSS as well. Figure 5B shows the dependence on the position of the dent. It has been found that a higher signal can be obtained when a particle adsorbs near the center of the membrane. An interesting feature of MSS is that it is more robust in position of the dent compared to cantilever-type sensors. In the case of cantilever-type sensors, a dent at $l=450 \mu \mathrm{m}$ causes $\sim 60$ times larger deflection than a dent at $l=50 \mu \mathrm{m}$. On the other hand, the signal caused by a dent at the center of MSS is not 10 times larger than the signal caused by a dent at $50 \mu \mathrm{m}$ from the edge. This result indicates that MSS is more robust in the adsorption position of a particle compared to cantilever-type sensors. The dependence of $\Delta R_{\text {tot }} / R_{\text {tot }}$ on a dent depth $h$ is quite similar to the case of cantilever-type sensors; a higher sensing signal can be obtained for a deeper dent (Figure 5C).

$\Delta R_{\text {tot }} / R_{\text {tot }}$ for various $E$ and $\nu$ are plotted in Figure 6. Figure 6A shows $\Delta R_{\text {tot }} / R_{\text {tot }}$ has little dependence on $E$ below $10^{9}$ $\mathrm{Pa}$, while $\Delta R_{\text {tot }} / R_{\text {tot }}$ decreases with $E$ over $10^{9} \mathrm{~Pa}$. As shown in Figure 6B, Poisson's ratio does not significantly affect $\Delta R_{\text {tot }} / R_{\text {tot }}$ when $E$ is below $10^{9} \mathrm{~Pa}$. However, a higher Poisson's ratio results in a lower $\Delta R_{\text {tot }} / R_{\text {tot }}$ in the case of $E>10^{9} \mathrm{~Pa}$. The simulated $\Delta R_{\text {tot }} / R_{\text {tot }}$ as a function of thickness is plotted in Figure 6C. The behavior is similar to the case of cantilever-type sensors (Figure 4C). The receptor layer with a soft material $\left(E<10^{8}\right.$ $\mathrm{Pa}$ ) exhibits little dependence on thickness, while the signal drastically decreases with thickness for a hard material $(E>$ $\left.10^{8} \mathrm{~Pa}\right)$.

\section{DISCUSSION}

The presented results indicate that a thinner and softer receptor layer will lead to a larger signal for the purpose of the detection of particles. It is known that there is an optimum thickness which yields maximum deflection in the cases of two typical nanomechanical sensing systems: the $2 \mathrm{D}$ stress applied homogeneously on the surface of a receptor layer, and the isotropic internal strain induced in a receptor layer (Yoshikawa, 2011; Yoshikawa et al., 2014). In contrast to such systems, an optimum thickness has not been found for any Young's moduli in the present simulation. This difference can be ascribed to the lack of the lateral stress enhancement effect in the case of particle adsorption. The effective stress induced on a sensing body is enhanced with increasing thickness of a receptor layer for the $2 \mathrm{D}$ stress or the isotropic internal strain because the lateral component of the force is applied further away from the neutral axis of the system. However, such effect for the present simulation is marginal because an effective force in the lateral direction is minute, hardly contributing to the deflection. Thus, the stiffening effect caused by the increase in thickness and/or Young's modulus becomes dominant, resulting in a smaller signal for thicker and stiffer receptor layer. The dependence on the position is found to be consistent with simple mechanics; a larger distance from a fixed-end provides a larger deflection in the cases of both cantilever-type and MSS.

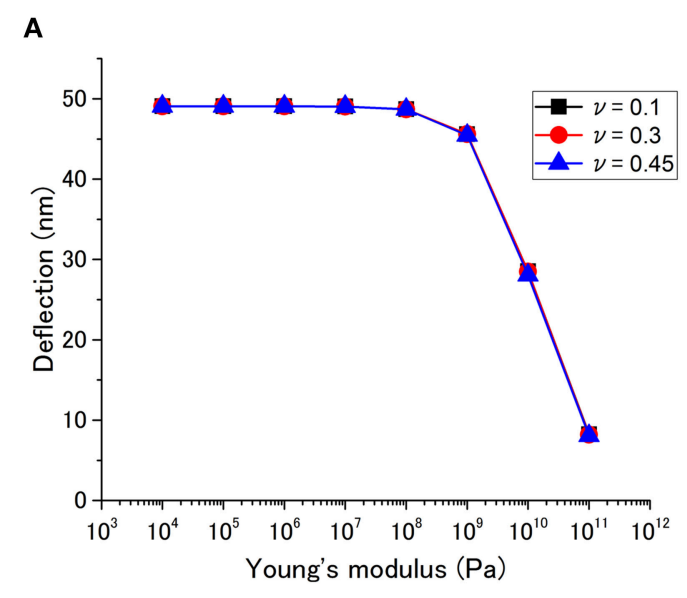

B
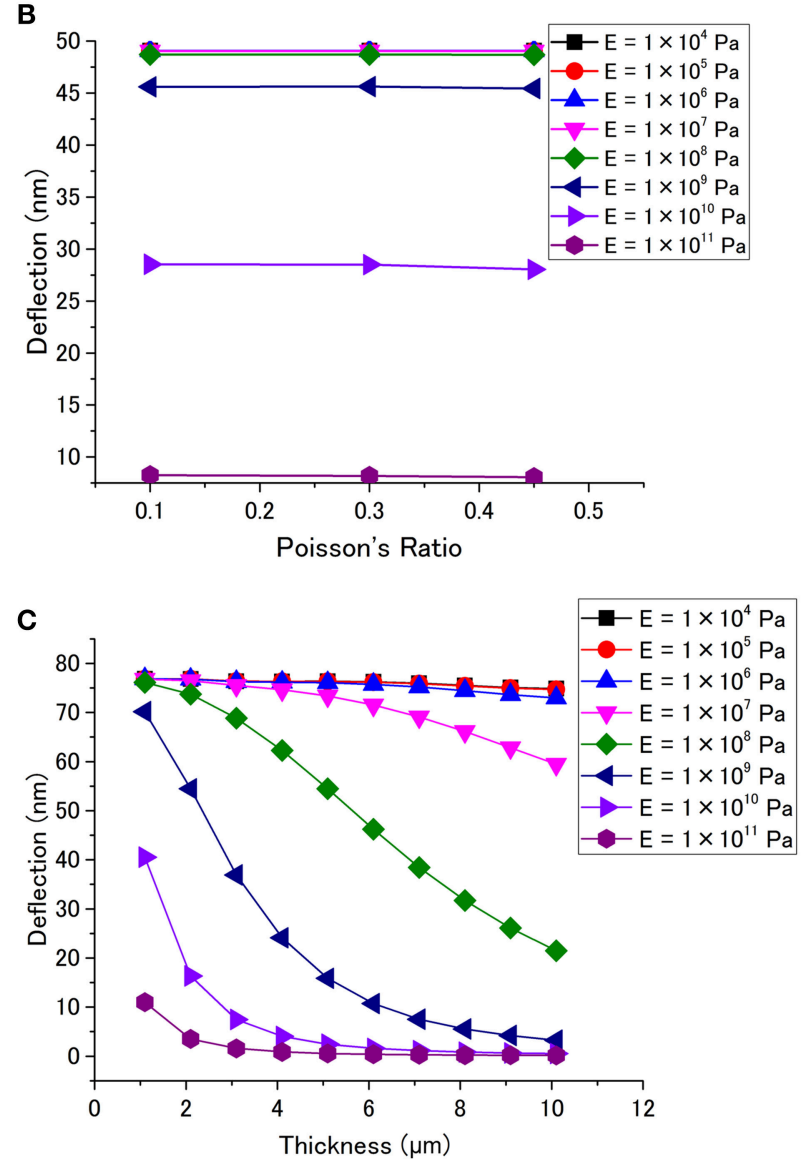

FIGURE 4 | Cantilever deflection as a function of (A) Young's modulus, and (B) Poisson's ratio. $\left(p=1 \times 10^{5} \mathrm{~Pa}, I=450 \mu \mathrm{m}, h=0.1 \mu \mathrm{m}, t=1\right.$ $\mu \mathrm{m})$ (C) Dependence of the deflection on the thickness for different Young's modulus $\left(p=1 \times 10^{5} \mathrm{~Pa}, /=450 \mu \mathrm{m}, h=0.1 \mu \mathrm{m}\right)$.

\section{Toward Virus Detection}

Based on these results, here we discuss a strategy to efficiently detect viruses with nanomechanical sensors. Several studies reported virus detection using nanomechanical sensors. These studies fall into two categories in terms of the operation 

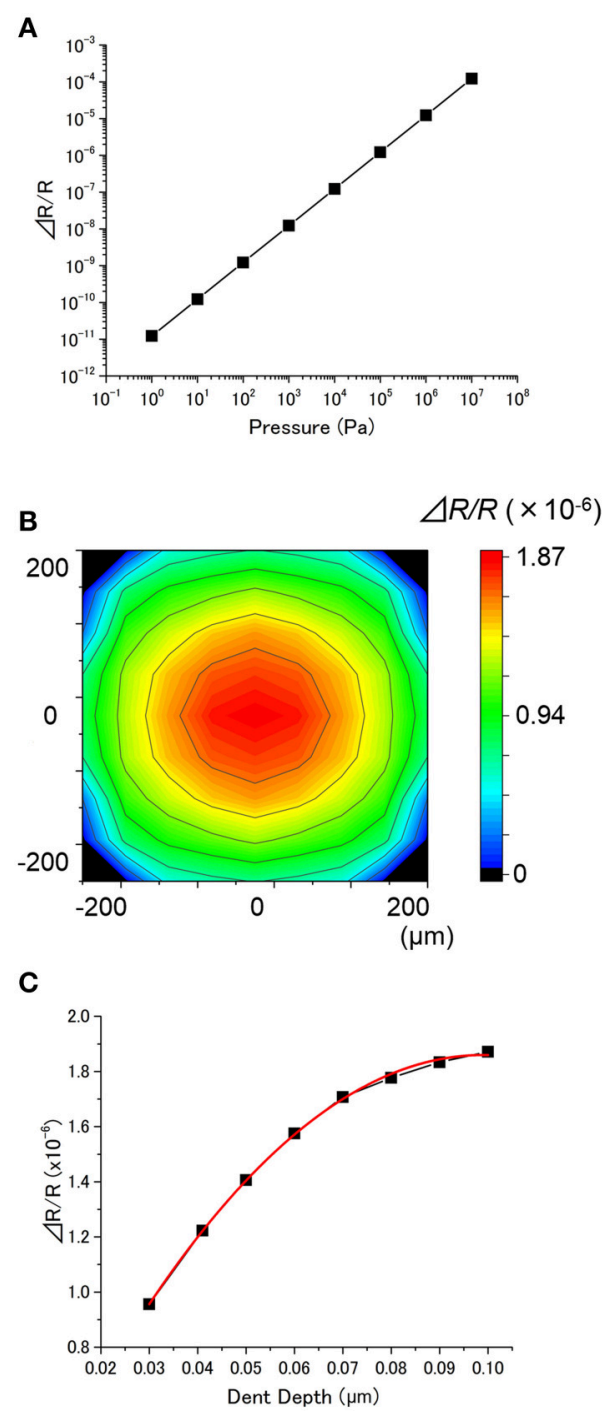

FIGURE 5 | (A) Dependence of $\Delta R / R$ on the applied pressure (the dent is at the center, $\left.h=0.1 \mu \mathrm{m}, t=1 \mu \mathrm{m}, E=1 \times 10^{9} \mathrm{~Pa}, v=0.4\right)$. (B) Dependence of $\Delta R / R$ on the position of the dent. The resultant relative resistance change is plotted as a color gradient $\left(p=1 \times 10^{5} \mathrm{~Pa}, h=0.1 \mu \mathrm{m}, t=1 \mu \mathrm{m}, E=\right.$ $\left.1 \times 10^{9} \mathrm{~Pa}, v=0.4\right)$. (C) Dependence of $\Delta R / R$ on the dent depth $\left(p=1 \times 10^{5}\right.$ $\mathrm{Pa}$, the dent is at the center, $\left.t=1 \mu \mathrm{m}, E=1 \times 10^{9} \mathrm{~Pa}, v=0.4\right)$. The plot is fitted with a quadratic function (red curve).

mode: dynamic mode and static mode. Nanomechanical sensors operated in dynamic mode detect viruses by their mass (more specifically, shifts in the resonant frequency; Johnson et al., 2006; Braun et al., 2009; Cha et al., 2009; Capobianco et al., 2010) while static mode operates based on the stress or strain (Gunter et al., 2003; Sreepriya and Hai-Feng, 2006; Alodhayb et al., 2013; Xu et al., 2014; Gorelkin et al., 2015; Kim et al., 2015). Thus, the FEA simulation in the present study can be adapted to the detection of viruses in static mode.

Previous studies revealed that the force caused by a virus attached to a cell is on the order of $10 \mathrm{pN}$ (Lee et al., 2006; Sieben et al., 2012; Tsai et al., 2015). Assuming that a virus

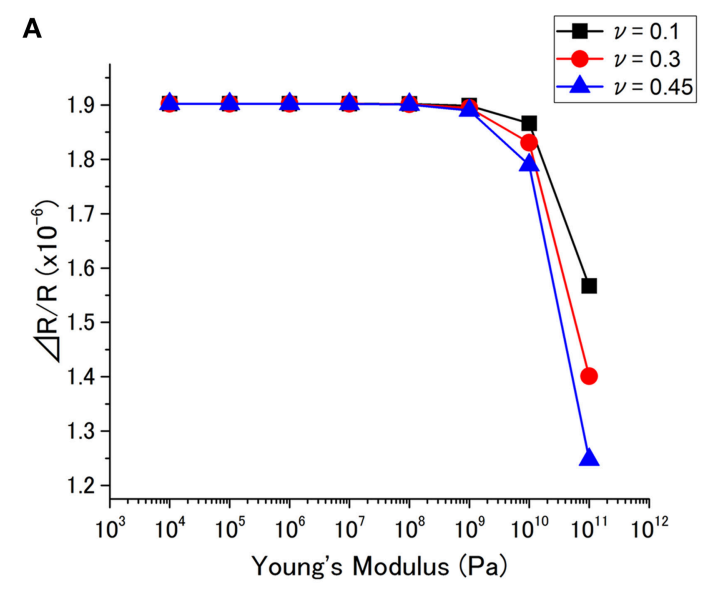

B
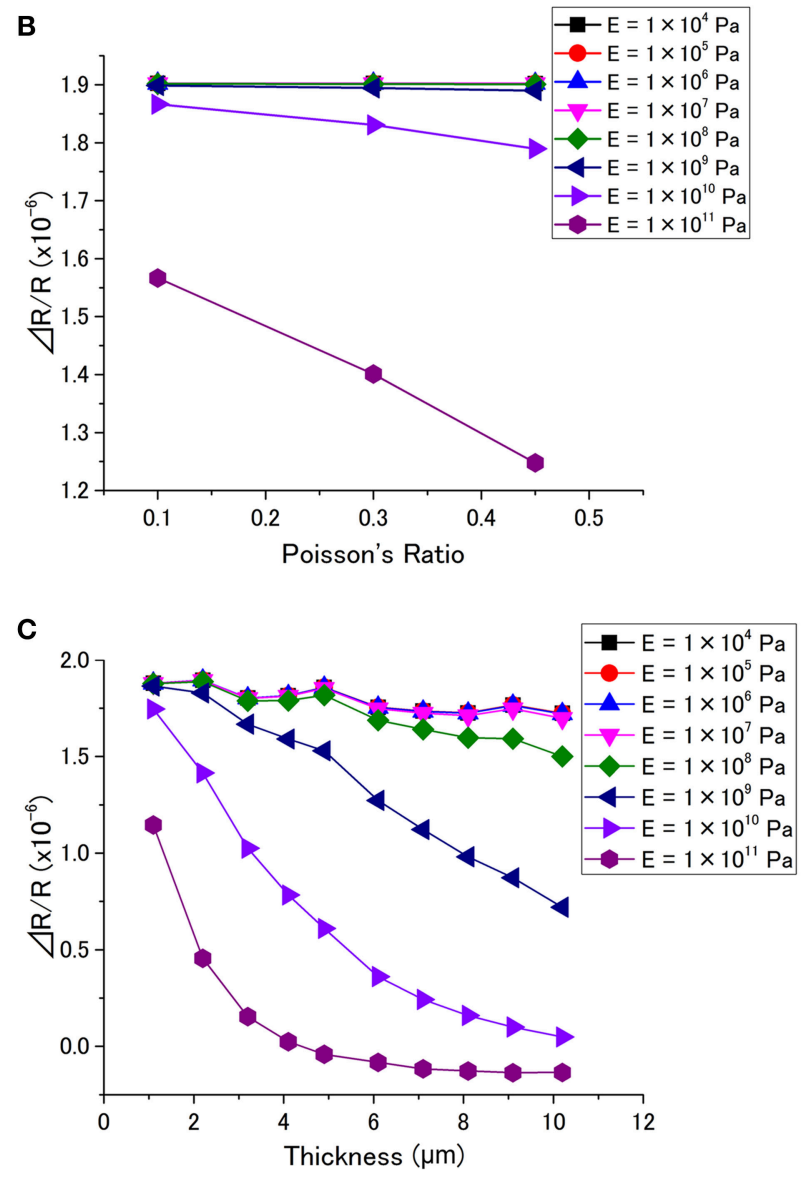

FIGURE $6 \mid \Delta R / R$ as a function of (A) Young's modulus, and (B) Poisson's ratio $\left(p=1 \times 10^{5} \mathrm{~Pa}\right.$, the dent is at the center, $h=0.1 \mu \mathrm{m}$, $\boldsymbol{t}=\mathbf{1} \boldsymbol{\mu} \mathbf{m})$. (C) Dependence of $\Delta R / R$ on the thickness for different Young's modulus $\left(p=1 \times 10^{5} \mathrm{~Pa}\right.$, the dent is at the center, $\left.h=0.1 \mu \mathrm{m}\right)$.

of $0.1 \mu \mathrm{m}$ in radius is embedded $0.1 \mu \mathrm{m}$ deep into a cell, the pressure applied to the interface is estimated to be $\sim 10^{3} \mathrm{~Pa}$. In the present simulations, the applied pressure $p$ is set at $1 \times 10^{5}$ $\mathrm{Pa}$ (Figures 3B-D, 4, 5B,C, 6), which is two orders of magnitude higher than the pressure caused by a virus. As the deflection 
and relative resistance changes of a cantilever-type sensor and MSS, respectively, are proportional to the applied pressure $p$, it is expected that the deflection/relative resistance change increases linearly with the number of adsorbed viruses on the receptor layer if the adsorbed positions of viruses are effectively regarded as constant. Accordingly, the presented simulations, in which $p$ is set at $1 \times 10^{5} \mathrm{~Pa}$, are comparable to a system where a hundred of viruses are attached to a specific position on the receptor layer. Considering that the typical detection limits for cantilever-type sensors and MSS are estimated as $\sim 1 \mathrm{~nm}$ and $\sim 10^{-6}$, respectively (Yoshikawa et al., 2011, 2012), a detectable signal can be obtained when $\sim 10^{2}$ of viruses adsorb near the free-end of a cantilever or the center of MSS with a receptor layer having optimal properties $\left(t=1 \mu \mathrm{m}, h=0.1 \mu \mathrm{m}, E<10^{8} \mathrm{~Pa}\right)$. To compare with typical nanomechanical sensing based on isotropic internal strain or surface stress, we also evaluated the corresponding detectable sensing signals. We performed FEA simulations on both a cantilever and an MSS which are coated with poly (methyl methacrylate; PMMA); a commonly used polymer as a receptor material, which has the Young's modulus and the Poisson's ratio of $3 \times 10^{9} \mathrm{~Pa}$ and 0.4 , respectively. The thickness was set at their optimum values: 2.5 and $8.0 \mu \mathrm{m}$ for the cantilever and the MSS, respectively (Yoshikawa, 2011; Yoshikawa et al., 2014). The minimum isotropic internal strain or surface stress which induces detectable signals (e.g., $1 \mathrm{~nm}$ deflection for the cantilever and $10^{-6}$ relative resistance change for the MSS) has been estimated as $10^{-9} \sim 10^{-8}$ or $10^{-5} \sim 10^{-4} \mathrm{~N} / \mathrm{m}$, respectively.

In terms of the receptor material, it has been confirmed that higher sensing signals can be obtained by using a material with low Young's modulus $\left(<10^{9} \mathrm{~Pa}\right)$. This is a favorable condition for practical applications because most biological materials which can interact with viruses have a low Young's modulus. For example, the Young's moduli of proteins and cells which are typically used for viral cultures are $10^{4} \sim 10^{9} \mathrm{~Pa}$ and $\sim 10^{4}$ $\mathrm{Pa}$, respectively. Another advantage of using soft materials is that signals are less affected by thickness, leading to higher reproducibility for virus detection. It should be noted that the force caused by the viruses may not be fully transmitted to the cantilever or MSS membrane in an actual measurement system if the receptor layer is thick and soft. Since viscoelastic properties are not taken into account in the present simulations, signals can be reduced as a result of stress relaxation within a receptor layer.

As living cells are the only media where viruses can be cultivated, there is a possibility that living cells are used as receptor materials. It is expected that selectivity will be enhanced depending on the cell types only if cells are stably maintained on nanomechanical sensors. However, the noise caused by cell migration is one of the biggest experimental issues. Bischofs et al. measured the surface stress induced by an endothelial cell and estimated it to be $\sim 2 \mathrm{mN} / \mathrm{m}$ (Bischofs et al., 2009). Our FEA simulations showed that a surface stress of $2 \mathrm{mN} / \mathrm{m}$ leads to a sensing signal comparable to adsorption of $\sim 10^{2}$ viruses on a receptor layer. Thus, the cellular force should give rise to a nonnegligible background noise. It is estimated that at least $10^{2} \sim 10^{3}$ of viruses are needed to obtain a detectable sensing signal when living cells are used as receptor materials.

Finally, let us compare the practical aspects between a cantilever and MSS. While the sensitivity of a cantilever sensor with an optical read-out is comparable to that of an MSS, a cantilever sensor requires a bulky instrumentation. In contrast, a compact measurement system can be realized with an MSS because of the chip-integrated piezoresistive electric sensing. Furthermore, MSS exhibits higher robustness to the adsorption position and in homogeneous coating (Loizeau et al., 2015). Thus, MSS seems to be a more favorable option for practical applications such as mobile environmental monitoring systems, point-of-care-testing (POCT) and primary screening for infectious diseases, etc. Although the sensitivity of MSS is not as high as that of enzyme-linked immunosorbent assay (ELISA), which can detect $\sim 10^{1}$ of viruses (Pineda et al., 2009), MSS is a promising candidate for a portable real-time virus detection device based on its simple detection mechanism and the easier experimental protocols.

\section{AUTHOR CONTRIBUTIONS}

GI, KS, and GY designed the simulation models. GI conducted the finite element analysis, and wrote the main paper. All authors discussed the results and implications, and commented on the manuscript at all stages.

\section{ACKNOWLEDGMENTS}

We express our appreciation to Mr. Masaki Anraku, graduate school of medicine, Yokohama City University, for his advice on the sensitivity of ELISA. We acknowledge Mr. Max Hamilton and Ms. Manasa Kaniselvan, University of Waterloo, for a valuable discussion. This research was supported by the World Premier International Research Center Initiative (WPI) on Materials Nanoarchitectonics (MANA).

\section{REFERENCES}

Aliabadi, A. A., Rogak, S. N., Bartlett, K. H., and Green, S. I. (2011). Preventing airborne disease transmission: review of methods for ventilation design in health care facilities. Adv. Prev. Med. 2011, 21. doi: 10.4061/2011/124064

Alodhayb, A., Brown, N., Saydur Rahman, S. M., Harrigan, R., and Beaulieu, L. Y. (2013). Towards detecting the human immunodeficiency virus using microcantilever sensors. Appl. Phys. Lett. 102, 173106. doi: 10.1063/1. 4803771

Barnes, J. R., Stephenson, R. J., Welland, M. E., Gerber, C., and Gimzewski, J. K. (1994). Photothermal spectroscopy with femtojoule sensitivity using a micromechanical device. Nature 372, 79-81. doi: 10.1038/372079a0

Bischofs, I. B., Schmidt, S. S., and Schwarz, U. S. (2009). Effect of adhesion geometry and rigidity on cellular force distributions. Phys. Rev. Lett. 103, 048101. doi: 10.1103/PhysRevLett.103.048101

Braun, T., (2009). Quantitative time-resolved measurement of membrane proteinligand interactions using microcantilever array sensors. Nat. Nano 4, 179-185. doi: $10.1038 /$ nnano. 2008.398 
Buchapudi, K. R., Huang, X., Yang, X., Ji, H.-F., and Thundat, T. (2011). Microcantilever biosensors for chemicals and bioorganisms. Analyst 136, 1539-1556. doi: 10.1039/c0an01007c

Capobianco, J. A., Shih, W.-H., Leu, J.-H., Lo, G. C.-F., and Shih, W. Y. (2010). Label free detection of white spot syndrome virus using lead magnesium niobate-lead titanate piezoelectric microcantilever sensors. Biosens. Bioelectron. 26, 964-969. doi: 10.1016/j.bios.2010. 08.004

Cha, B. H., (2009). Detection of Hepatitis B Virus (HBV) DNA at femtomolar concentrations using a silica nanoparticle-enhanced microcantilever sensor. Biosens. Bioelectron. 25, 130-135. doi: 10.1016/j.bios.2009. 06.015

Després, V. R., Huffman, J. A., Burrows, S. M., Hoose, C., Safatov, A. S., Buryak, G., et al. (2012). Primary biological aerosol particles in the atmosphere: a review. Tellus B 64:15598. doi: 10.3402/tellusb.v64i0.15598

Gimzewski, J. K., Gerber, C., Meyer, E., and Schlittler, R. R. (1994). Observation of a chemical reaction using a micromechanical sensor. Chem. Phys. Lett. 217, 589-594. doi: 10.1016/0009-2614(93)E1419-H

Gorelkin, P. V., (2015). Synthetic sialylglycopolymer receptor for virus detection using cantilever-based sensors. Analyst 140, 6131-6137. doi: 10.1039/C5AN01102G

Gunter, R. L., Delinger, W. G., Manygoats, K., Kooser, A., and Porter, T. L. (2003). Viral detection using an embedded piezoresistive microcantilever sensor. Sens. Actuators A Phys. 107, 219-224. doi: 10.1016/S0924-4247(03) 00379-0

Hosokawa, N., Nakamichi, M., Honda, N., Shiba, K., and Yoshikawa, G. (2014). "Antibody-based protein detection using nanomechanical Membranetype Surface Stress Sensor (MSS)," in 11th International Workshop on Nanomechanical Sensing Workshop (NMC2014) (Madrid).

Johnson, L., Gupta, A. K., Ghafoor, A., Akin, D., and Bashir, R. (2006). Characterization of vaccinia virus particles using microscale silicon cantilever resonators and atomic force microscopy. Sens. Actuators B Chem. 115, 189-197. doi: 10.1016/j.snb.2005.08.047

Kim, H. H., (2015). Highly sensitive microcantilever biosensors with enhanced sensitivity for detection of human papilloma virus infection. Sens. Actuators B Chem. 221, 1372-1383. doi: 10.1016/j.snb.2015. 08.014

Kim, K.-H., Kabir, E., and Kabir, S. (2015). A review on the human health impact of airborne particulate matter. Environ. Int. 74, 136-143. doi: 10.1016/j.envint.2014.10.005

Lee, J. W. M., Chu, J. J. H., and Ng, M. L. (2006). Quantifying the specific binding between West Nile virus envelope domain III protein and the cellular receptor alpha V beta 3 integrin. J. Biol. Chem. 281, 1352-1360. doi: 10.1074/jbc.M506614200

Loizeau, F., (2015). Comparing membrane- and cantilever-based surface stress sensors for reproducibility. Sens. Actuators A Phys. 228, 9-15. doi: 10.1016/j.sna.2015.02.039

Nemmar, A., Holme, J. A., Rosas, I., Schwarze, P. E., and Alfaro-Moreno, E. (2013). Recent advances in particulate matter and nanoparticle toxicology: a review of the in vivo and in vitro studies. Biomed Res. Int. 2013:279371. doi: 10.1155/2013/ 279371
Pineda, M. F., (2009). Rapid specific and label-free detection of porcine rotavirus using photonic crystal biosensors. IEEE Sens. J. 9, 470-477. doi: 10.1109/JSEN.2009.2014427

Sieben, C., (2012). Influenza virus binds its host cell using multiple dynamic interactions. Proc. Natl. Acad. Sci. U.S.A. 109, 13626-13631. doi: 10.1073/pnas.1120265109

Sreepriya, V., and Hai-Feng, J. (2006). Detection of feline coronavirus using microcantilever sensors. Meas. Sci. Technol. 17, 2964. doi: 10.1088/09570233/17/11/015

Stoney, G. G. (1909). The tension of metallic films deposited by electrolysis. Proc. R. Soc. Lond. A Math. Phys. Eng. Sci. 82, 172-175. doi: 10.1088/09570233/17/11/015

Thundat, T., Warmack, R. J., Chen, G. Y., and Allison, D. P. (1994). Thermal and ambient-induced deflections of scanning force microscope cantilevers. Appl. Phys. Lett. 64, 2894-2896. doi: 10.1063/1.111407

Tsai, B.-Y., Chen, J.-Y., Chiou, A., and Ping, Y.-H. (2015). Using optical tweezers to quantify the interaction force of dengue virus with host cellular receptors. Microsc. Microanal. 21, 219-220. doi: 10.1017/S14319276 15001890

Xu, D., Liu, L., Guan, J., Xu, J., Wang, T., Qin, A., et al. (2014). Label-free microcantilever-based immunosensors for highly sensitive determination of avian influenza virus H9. Microchim. Acta 181, 403-410. doi: 10.1007/s00604$013-1129-\mathrm{x}$

Yoshikawa, G. (2011). Mechanical analysis and optimization of a microcantilever sensor coated with a solid receptor film. Appl. Phys. Lett. 98, 173502. doi: $10.1063 / 1.3583451$

Yoshikawa, G., Akiyama, T., Gautsch, S., Vettiger, P., and Rohrer, H. (2011). Nanomechanical membrane-type surface stress sensor. Nano Lett. 11, 1044-1048. doi: 10.1021/nl103901a

Yoshikawa, G., Lee, C. J. Y., and Shiba, K. (2014). Effects of coating materials on two dimensional stress-induced deflection of nanomechanical sensors. J. Nanosci. Nanotechnol. 14, 2908-2912. doi: 10.1166/jnn.2014.8604

Yoshikawa, G., Akiyama, T., Loizeau, F., Shiba, K., Gautsch, S., Nakayama, T., et al. (2012). Two dimensional array of piezoresistive nanomechanical MembraneType Surface Stress Sensor (MSS) with improved sensitivity. Sensors 12, 15873-15887. doi: 10.3390/s121115873

Conflict of Interest Statement: The authors declare that the research was conducted in the absence of any commercial or financial relationships that could be construed as a potential conflict of interest.

The reviewer TY and handling Editor declared a current collaboration and the handling Editor states that the process nevertheless met the standards of a fair and objective review.

Copyright (c) 2016 Imamura, Shiba and Yoshikawa. This is an open-access article distributed under the terms of the Creative Commons Attribution License (CC BY). The use, distribution or reproduction in other forums is permitted, provided the original author(s) or licensor are credited and that the original publication in this journal is cited, in accordance with accepted academic practice. No use, distribution or reproduction is permitted which does not comply with these terms. 